Available online on 15.03.2020 at http://jddtonline.info
Open Access to Pharmaceutical and Medical Research
unrestricted non-commercial use, provided the original work is properly cited

open Access

Research Article

\title{
Pharmacophore and Molecular Docking-Based Virtual Screening of B-Cell Lymphoma 2 (BCL 2) Inhibitor from Zinc Natural Database as Anti-Small Cell Lung Cancer
}

\author{
Fauzan Zein Muttaqin 1*; Dina Kharisma 1; Aiyi Asnawi ${ }^{2}$; and Fransiska Kurniawan ${ }^{2}$ \\ ${ }^{1}$ Bandung School of Pharmacy, Bandung, West Java, 40161, Indonesia \\ ${ }^{2}$ School of Pharmacy, Bandung Institute of Technology, Bandung, West Java, 40132, Indonesia
}

\begin{abstract}
Cancer is a disease involving genetic factors in its pathogenesis. The increase of cell survival as a result of genetic changes, which prevent apoptosis such as Bcl2 (B-cell lymphoma-2) activation, will cause the tumor to grow. The overexpression of Bcl2 in small cell lung cancer should be inhibited. This study aims to screen natural products that can inhibit Bcl2 overexpression in lung cancer using pharmacophore- and molecular docking-based virtual screening to ZINC Natural Product database. The validation of pharmacophore-based virtual screening to the three features of the pharmacophore model ( 2 hydrophobic interactions and 1 hydrogen bond donor) showed that the AUC, EF, Se, Sp, ACC, and GH values were $0.57,3.8,0.101,0.957,0.936$, and 0.149 , respectively. On the other hand, the validation of molecular docking-based virtual screening showed that the RMSD values of Vina Wizard and AutoDock Wizard were $1.3 \AA$ and $1.9 \AA$, respectively. The pharmacophore model virtual screening first obtained 6,615 compounds, and then the molecular docking-based virtual screening finally gained 255 compounds whose values of $\Delta \mathrm{G}$ and Ki were lower than those of the native ligand. It was concluded that the virtual screening could yield as many as 255 potential anti-lung cancer drug candidates.
\end{abstract}

Keywords: B-cell lymphoma 2 inhibitors, molecular docking, pharmacophore modeling, virtual screening

Article Info: Received 10 Jan 2020; Review Completed 21 Feb 2020; Accepted 29 Feb 2020; Available online 15 March 2020

Cite this article as:

Muttaqin FZ, Kharisma D, Asnawi A, Kurniawan F, Pharmacophore and Molecular Docking-Based Virtual Screening of BCell Lymphoma 2 (BCL 2) Inhibitor from Zinc Natural Database as Anti-Small Cell Lung Cancer, Journal of Drug Delivery and Therapeutics. 2020; 10(2):143-147 http://dx.doi.org/10.22270/jddt.v10i2.3923

*Address for Correspondence:

Fauzan Zein Muttaqin, Bandung School of Pharmacy, Bandung, West Java, 40161, Indonesia

\section{INTRODUCTION}

Cancer is a disease that involves genetic factors in its pathogenesis. The process of cell division is uncontrolled since the genes that regulate cell growth have been damaged. The activation of several oncogenes results in the deregulation of cell proliferation, which is often associated with apoptosis; and, the result of increased apoptosis prevents the tumor to enlarge. However, increased cell survival as a result of genetic changes prevents apoptosis i.e. activation of B-cell lymphoma (Bcl2), thus, causing tumors to grow larger 1.

Cancer is the leading cause of death worldwide, accounting for 8.8 million deaths in 2015. The most common causes of death include lung, liver, colorectal, stomach, and breast cancers ${ }^{2}$. In Indonesia, the prevalence of cancer has been quite high, with approximately 1.4 per 1,000 population, or 347,000 people 3 , and lung cancer had the highest percentage of new cases of $23.1 \%$ and was the highest cause of death 4 .
$\mathrm{Bcl} 2$ is a negative regulator of cell death and plays an important role in apoptosis regulation. In small cell lung cancer, overexpression occurs in $\mathrm{Bcl} 2$, and thus, $\mathrm{Bcl} 2$ activity must be inhibited. Several Bcl2 inhibitors do not improve the results of therapy in small cell lung cancer patients ${ }^{5}$. Therefore, it is necessary to search for new active compounds that are projected to be able to inhibit Bcl2 overexpression.

In the discovery of new drugs, drug testing is not only carried out in vivo or in vitro but also in silico or by computer simulations 6 . Virtual screening is a computational method that reduces the number of chemical compounds that will be identified experimentally in a faster time ${ }^{7}$. The method of pharmacophore-based virtual screening is now commonly used as part of a more complex workflow in drug discovery and has been successful and widely applied 8. In this study, pharmacophore- and molecular docking-based virtual screening were used on Zinc natural product database 
compounds to seek candidates for $\mathrm{Bcl} 2$ inhibitor compounds as anti-lung cancer. The study first employed the pharmacophore-based virtual screening and then followed by the docking-based virtual screening.

\section{RESEARCH METHODS}

\section{Hardware}

The hardware used in this study was a computer unit with the specifications of Windows 8.1 Pro 64-bit operating system, Intel® CoreTM i7-4790 CPU @ $3.60 \mathrm{GHz}$ 8 (CPUs), 16 GB of RAM DDR3 memory, and 4 GB 128-bit VGA dedicated.

\section{Protein Structure Preparation of Bcl2}

The structure of the target macromolecule (Bcl-2 inhibitor) (GDP ID: 3SPF) ${ }^{9}$ was obtained from the PDB website (www.rcsb.org).

\section{Ligand Preparation}

The database of the test compounds used for virtual screening was the database of natural product compounds gained from the ZINC database. The number of natural products in the ZINC database was 151,837 compounds. Each natural product database was grouped based on the origin of the country and contains natural compounds derived from plants. The ZINC database consisted of 12 databases, including AfroDB Natural Product, AnalytiCon Discovery NP, Herbal Ingredients InVivo Metabolism, Herbal Ingredients Targets, IBScreen NP, Indofine Natural Products, NPACT Database, Nubbe Natural Products, Princeton NP, Specs Natural Products, TCM Database Taiwan, and UEFS Natural Products.

The active compounds acting as a positive control in the validation of the virtual screening were obtained from the website https://www.ebi.ac.uk/chembl/. These active compounds were then re-selected following the standard value (IC 50 ). Afterward, the $\mathrm{IC}_{50}$ values were sorted out by using Microsoft Excel, with the provision that the $\mathrm{IC}_{50}$ values should have been smaller than $10,000 \mathrm{nM}$, yielding as many as 40 compounds.

On the other hand, the decoy compounds used as negative control were those inactive compounds acquired by searching through Decoy Finder 2.0 software ${ }^{10}$. The ligand datasets known to be active Bcl-2 inhibitors were inserted in the Decoy Finder and later stored in the same folder for further processing. There were 1,440 decoy compounds generated from the process.

\section{Pharmacophore-based Virtual Screening}

The pharmacophore modeling utilized the LigandScout 4.0 software 11 . First, the native ligand optimization of the $\mathrm{Bcl} 2$ receptor was conducted and then stored in a 3D format. The pharmacophore of the optimized native ligand was created and also validated. The validation was carried out by applying the overall pharmacophore features of the native ligand against the active and decay compounds. The validation was performed by observing the values of the hit compounds and the ROC curves which contained the values of AUC of more than 0.5 and of EF of over 1.08 and also by calculating other classic enrichment validation parameters such as Se values 12,13, Sp values 13, ACC values 14,15,16, Ya values ${ }^{17}$, and $\mathrm{GH}$ values ${ }^{18}$. The validated pharmacophore models were used for the virtual screening of compounds in the ZINC Natural Product Database.

\section{Molecular Docking-based Virtual Screening (PyRx 8.0)}

Molecular docking was carried out by using the Pyrx 8.0 software 19 together with Vina Wizard and AutoDock Wizard. The macromolecular structure of the $\mathrm{Bcl} 2$ receptor was downloaded from the Protein Data Bank with PDB ID 3SPF. The active site of the protein was made following the native ligand binding side of $\mathrm{Bcl} 2$ with a grid width of $40 \times 40 \times 40$ on the XYZ axis with a grid point spacing of $0.375 \AA$ A. Molecular docking applied as many as 10 repetitions. Other parameters were used according to the default value. The results of the validation of the docking method are declared valid if the RMSD value is less than $2 \AA 20$.

This virtual screening validation was done by using the parameters relevant to those in the molecular docking of active and decoy compounds. Validation was carried out by taking into account the validation parameter values as those in the pharmacophore validation. The ROC curves were created on the website http://rocr.bioinf.mpisb.mpg.de/ ${ }^{21}$. The validated model was then employed for virtual screening of compounds of the ZINC natural product database from the previous screening. The result was further examined by virtual docking-based screening.

\section{RESULTS AND DISCUSSION}

Virtual screening (VS) is a computational filter to reduce the size of a chemical library that will be identified experimentally and offers opportunities to quickly reduce the time and effort associated with identification ${ }^{7}$. VS is described as the use of high-performance computational calculations in analyzing a database of many chemical compounds in order to identify the probability of drug candidates ${ }^{22}$. There are two fundamental approaches to virtual screening: the ligand-based approach and the receptor-based approach. The ligand-based approach aims to identify molecules with physical and chemical similarities (pharmacophore-based, descriptor-based) against identified ligands that tend to interact with targets. The structure-based pharmacophore modeling approach uses information about protein-ligand interactions obtained from experimental 3D structures in order to produce a model 23 .

\section{Pharmacophore-based Virtual Screening}

Prior to virtual screening, the validation of the pharmacophore features was employed first. The target was $\mathrm{Bcl} 2$ receptor (GDP code: $3 \mathrm{SPF}$ ) with native ligand 4(4-chlorophenyl)-1-[(3S) -3,4-dihydroxybutyl]-N-[3-(4methylpiperazin-1-yl) propyl] -3-phenyl-1H-pyrrole-2carboxamide $\left(\mathrm{C}_{29} \mathrm{H}_{37} \mathrm{Cl} \mathrm{N}_{4} \mathrm{O}_{3}\right)$ with a resolution of $1.7 \AA$. After optimization of the ligand bound to the $\mathrm{Bcl} 2$ receptor, the pharmacophore features from the ligand were generated. Only four pharmacophore features were obtained including 3 hydrophobic interactions and 1 hydrogen bond donor (Figure 1a.). These pharmacophore features were then validated with active and decoy datasets (Table 1). The first and second models gained were good because their AUC and EF values met the requirements. A good AUC value has $\mathrm{AUC} \geq 0.5$, a good $\mathrm{EF}$ value has $E F \geq 0.1$, a good $S e$ value equals to 1 , a good $S p$ value equals to 1 , and for the $\mathrm{GH}$ value, the higher the value, the better 8 . Therefore, by looking at the value of these classic enrichment parameters, the model one was created and used as a virtual pharmacophore model for screening the natural product database. This first model consisted of 3 pharmacophore features used later for virtual screening: 2 hydrophobic interactions and 1 hydrogen bond donor. 
Table 1 Validation of pharmacophore features

\begin{tabular}{cccccccc}
\hline Model & AUC 100\% & EF 100\% & Se & Sp & ACC & Ya & GH \\
\hline 1 & 0.57 & 3.8 & 0.101 & 0.957 & 0.936 & 0.175 & 0.149 \\
2 & 0.67 & 2.6 & 0.069 & 0.832 & 0.809 & 0.45 & 0.029 \\
3 & 0.48 & 0.5 & 0.014 & 0.901 & 0.878 & 0.05 & 0.021 \\
4 & 0.36 & 0 & 0.012 & 0.535 & 0.526 & 0.2 & 0.082 \\
5 & 0.49 & 0 & 0 & 0.982 & 0.955 & 0 & 0 \\
\hline
\end{tabular}

$A U C>0.5 ; E F>1 ; S e=1 ; S p=1 ; G H=$ the higher the value, the better

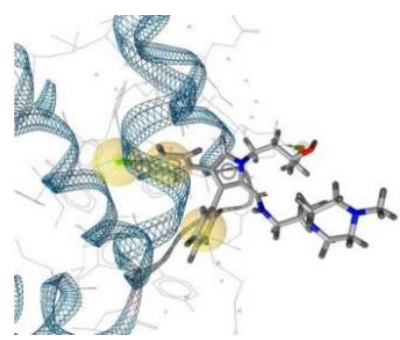

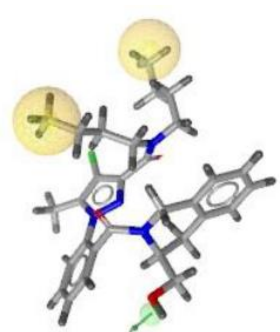

b

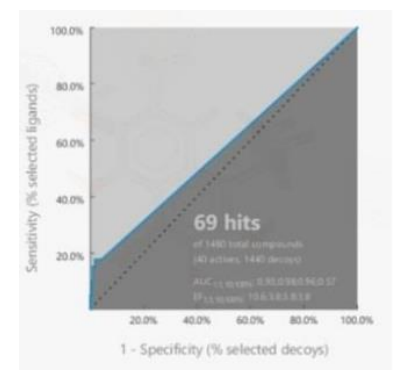

C

Figure 1. Pharmacophore features of native ligand (a), validated pharmacophore features (b), and ROC curves for the first model (c).

Here, the natural product compounds obtained from the site http://www.zinc.docking.org were firstly screened consisting of 12 databases with a total of 151,837 compounds. The virtual screening utilized the model one, as the validated pharmacophore model, resulting in 6,615 compounds. However, the outputs were still considered too many candidates; thus, further screening was needed to obtain fewer compounds.

\section{Molecular Docking-based Virtual Screening}

The next stage of the filtering process involved docking each molecule in the database into the target binding area. The docking process comprised sampling the coordinate space of the binding location and printing every possible ligand pose, which was later taken as the predicted binding mode for the compound ${ }^{24}$. Validation of the docking method was applied by re-docking the native ligand and $\mathrm{Bcl} 2$ target receptor.

The molecular docking with the test compounds using PyRx software was divided into 2 phases. Phase one employed Vina Wizard while phase two employed AutoDock Wizard. The re-docking processes with both Vina Wizard and Autodock Wizard were done in PyRx 8.0 software. Grid Box settings used for the Vina Wizard included X: 19.09, Y: 23.64, and Z: 21.33, whereas Grid Spacing used 1.0 ̊̊ with Grid Center X: 29.54, Y: 13.13, and Z: -21.68 . Meanwhile, those for Autodock Wizard included Grid Box X: 72, Y: 67, and Z: 68, and Grid Spacing of $1.0 \AA$ with Grid Center X: 28.94, Y: 10.29, and Z: -22.49 . The RSMD values for Vina Wizard and Autodock Wizard were $1.3 \AA$ and $1.99 \AA$ Å, respectively, and the docking method used was declared valid.

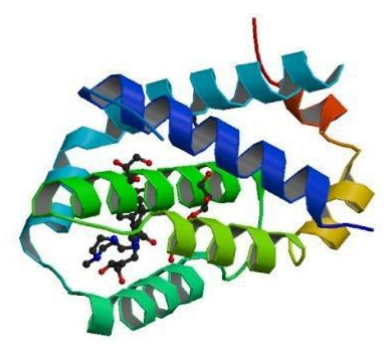

a

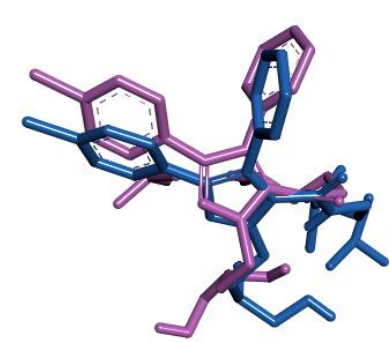

b

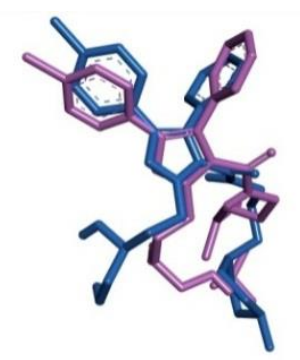

C

Figure 2 Visualization of Bcl 2 receptor (PDB code) (a) re-docking results with Vina Wizard (b), and re-docking results with Autodock Wizard (c) to validate the docking method.

Note: pink for the native ligand and blue for the ligand of re-docking 


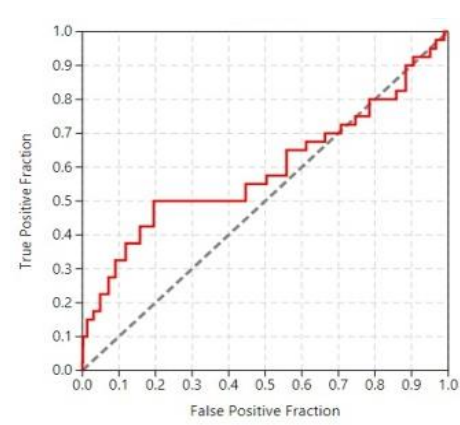

(a)

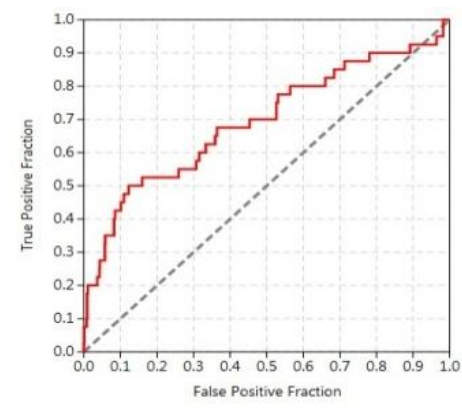

(b)

Figure 3 ROC curves of validation results of virtual screening method with Vina Wizard (a) and Autodock Wizard (b) for virtual validation of docking-based screening.

Note: $A$ total of 1,480 compounds with 40 active and 1,440 inactive compounds (decoys)

Further, virtual screening was carried out by using PyRx, and then virtual screening validation by using active and decoy compounds. Validation parameters for the Vina Wizard were AUC and EF values of 0.592 and 37.0, respectively, while those for the Autodock Wizard were AUC and EF values of 0.693 and 37.0, respectively. These findings indicated that the virtual screening validation in both the Vina Wizard and Autodock Wizard were valid enough since they met the requirements; therefore, screening could be carried out for the test compounds.

The screening results obtained at the Vina Wizard were 2,473 compounds, whose binding energy values were smaller than native ligand. These results were further screened by using the AutoDock Wizard, yielding 255 test compounds (Table 2).

\section{Identification of the best test compounds}

The screening results of the test compounds obtained were further analyzed in order to discover the best compounds. This analysis could be seen from the compounds with the smallest binding energy values, aside from the values of the inhibition constant. The values of binding energy and inhibition constant which were lower than the native ligand indicated that the binding strength between the ligands from the screening results and the receptor was better. The values of binding energy yielded 255 compounds, and then this result was later sorted out according to the values of the inhibition constant. In the native ligand, the inhibition constant value obtained was $128.81 \mu \mathrm{M}$ whereas these 255 compounds had smaller inhibition constant values. The smallest value of the binding energy of the inhibition constant earned by the ligand with the code tc259, having a BE value of -11.02 $\mathrm{kcal} / \mathrm{mol}$ and $\mathrm{Ki}$ of $8.33 \mathrm{nM}$. Another analysis performed was by using ligand interactions between amino acid residues of $\mathrm{Bcl} 2$ and test compounds (Figure 3). In the native ligand, there were hydrogen bonds on amino acid residues GLU129, making this a reference for obtaining the candidate compounds that interacted with the $\mathrm{Bcl} 2$ receptor. The interactions occurring between the ligands and the $\mathrm{Bcl} 2$ receptor included hydrogen bond, hydrophobic interaction, and Van der Waals interaction.

Table 2. Summary of the screening results of zinc natural product compounds

\begin{tabular}{llcccc}
\hline No. Database & $\begin{array}{c}\text { Zinc Natural } \\
\text { Product }\end{array}$ & $\begin{array}{c}\text { Pharmacophore- } \\
\text { based VS }\end{array}$ & \multicolumn{2}{c}{ Docking-based VS } \\
\cline { 4 - 5 } 1. & Afronp & 884 & 66 & 21 & 4 \\
2. & Acdiscnp & 11,217 & 786 & 54 & 28 \\
3. & Himnp & 652 & 36 & 20 & 10 \\
4. & Hitnp & 801 & 29 & 14 & 8 \\
5. & Indofinenp & 142 & 8 & 0 & 0 \\
6. & Ibsnp & 84,099 & 2,095 & 1,199 & 81 \\
7. & Npactnp & 1,421 & 94 & 19 & 16 \\
8. & Nubbenp & 584 & 28 & 12 & 31 \\
9. & Princetonnp & 14,084 & 312 & 38 & 4 \\
10. & Specsnp & 1,488 & 42 & 6 & 58 \\
11. & Tcmnp & 35,993 & 3,105 & 1,081 & 5 \\
12. & Uefsnp & 472 & 14 & 9 & 255 \\
\hline Total & & 151,837 & 6,615 & 2,473 & \\
\hline
\end{tabular}




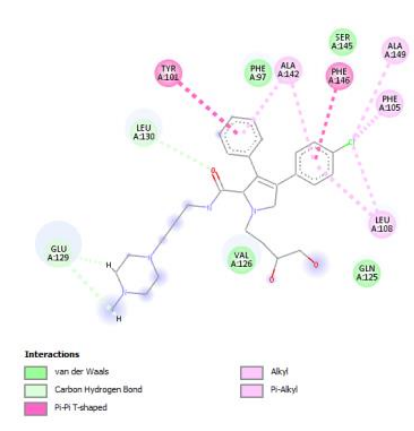

(a)

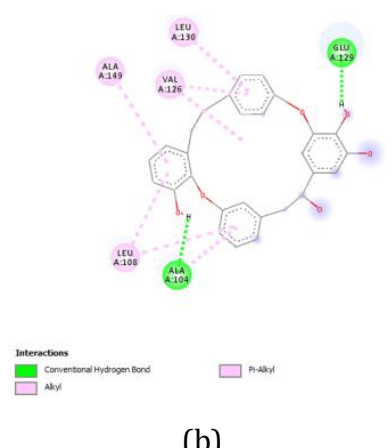

(b)

Figure 4 Interactions of (a) native ligand of re-docking results (b) test ligands with Bcl2 receptor

\section{CONCLUSIONS}

The pharmacophore-based virtual screening involving hydrophobic interactions and hydrogen bonds was able to eliminate approximately $85.64 \%$ of compounds. Further, the docking-based virtual screening was capable of diminishing about $96.15 \%$ of compounds predicted to inhibit Bcl2 protein overexpression. The interactions between the best test compounds and the $\mathrm{Bcl} 2$ receptor included Van der Waals interaction, hydrogen bond, and hydrophobic interaction.

\section{ACKNOWLEDGMENTS}

Authors thank the Center for Research and Community Services, Bandung School of Pharmacy, for the internal research fund.

\section{REFERENCES}

1. Engelberts PJ, Voorhorst M, Schuurman J, van Meerten T, Bakker JM, Vink T, et al., Type I CD20 Antibodies Recruit the B Cell Receptor for Complement-Dependent Lysis of Malignant B Cells, J Immunol, 2016, December 15, 2016, 197 (12): 48294837.

2. WHO, Media Center: Fact Sheet Cancer, 2017. Available in http://www.who.int/mediacentre/factsheets/fs297/en/ accessed on Oct 15, 2017

3. Ministry of Health of the Republic of Indonesia, Hasil Riskesdas 2013 [Results of Basic Health Research of 2013], 2013: 85-87. Accessed on October 14, 2017

4. Data and Information Center, Ministry of Health of the Republic of Indonesia, 2015. Available at: http://www.pusdatin.kemkes.go.id/folder/view/01/structur e-publikasi-pusdatin-profil-kesehatan.html [accessed 02 July 2017]

5. Semenova EA, Nagel R, Berns A, Origins, genetic landscape, and emerging therapies of small cell lung cancer, Cold Spring Harbor Laboratory Press. Genes \& Development, 2015, 29: 1447-1462.

6. Muttaqin FZ, Fakih TM, Muhammad HN, Molecular Docking, Molecular Dynamics, And In Silico Toxicity Prediction Studies Of Coumarin, N-Oxalylglycine, Organoselenium, Organosulfur, And Pyridine Derivatives As Histone Lysine Demethylase Inhibitors, Asian Journal of Pharmaceutical and Clinical Research, 2017, 10(12): 212-215.

7. Yadav M, Gurmith S, Virtual Screening of Ligand molecules for target protein CYP26A1 by using AutoDock-Vina, IJIRSET, 2013, 2(9): 4917.

8. Braga $\mathrm{RC}$, Andrade $\mathrm{CH}$, Assessing the performance of $3 \mathrm{D}$ pharmacophore models in virtual screening: how good are they?, 2013, 13(9):1127-38.
9. Zhou H, Chen J, Meagher JL, Yang CY, Aguilar A, Liu L., et al., Design of Bcl-2 and Bcl-xL Inhibitors with Subnanomolar Binding Affinities Based upon a New Scaffold. J.Med.Chem., 2012, 55: 4664-4682.

10. Adrià CM, Garcia-Vallvé $\mathrm{S}$, Pujadas G, DecoyFinder, a tool for finding decoy molecules, J Cheminform. 2012, 4(1): 2 .

11. Wolber G, Langer T, LigandScout: 3-D pharmacophores derived from protein-bound ligands and their use as virtual screening filters, J Chem Inf Model, 2005, 45(1): 160-169.

12. Langer $\mathrm{T}$, Hoffmann RD, Pharmacophores and Pharmacophore Searches; 1st ed.; Wiley-VCH Verlag GmbH \& Co. KGaA: Weinheim, FRG, 2006, 395.

13. Triballeau N, Acher F, Brabet I, Pin JP, Bertrand HO, Virtual screening workflow development guided by the "receiver operating characteristic" curve approach. Application to highthroughput docking on metabotropic glutamate receptor subtype 4, J. Med. Chem., 2005, 48: 2534-47.

14. Gao H, Williams C, Labute P, Bajorath J, Binary quantitative structure-activity relationship (QSAR) analysis of estrogen receptor ligands. J. Chem. Inf. Comput. Sci., 1999, 39: 164-8.

15. Jacobsson M, Lidén P, Stjernschantz E, Boström H, Norinder U, Improving structure-based virtual screening by multivariate analysis of scoring data, J. Med. Chem., 2003, 46: 5781-9.

16. Shepherd AJ, Gorse D, Thornton JM, Prediction of the location and type of beta-turns in proteins using neural networks, Protein Sci., 1999, 8: 1045-55.

17. Guner OF, Pharmacophore Perception, Development, and Use in Drug Design, 1st ed.; Intl Univ Line: La Jolla, 2000, 560.

18. Kurogi Y, Guner OF, Pharmacophore modeling and threedimensional database searching for drug design using catalyst, Curr. Med. Chem., 2001, 8: 1035-55.

19. Dallakyan S, Olson AJ, Small-Molecule Library Screening by Docking with PyRx. Methods Mol Biol., 2015, 1263:243-50

20. Morris MG, Ruth H, Lindstrom W, Sanner MF, Belew RK, Goodsell DS, et al., AutoDock4 and AutoDockTools4: Automated Docking with Selective Receptor Flexibility, J. Comput. Chem., 2009, 30: 2785-2791.

21. Sing T, Sander O, Beerenwinkel N, Lengauer T, ROCR visualizing classifier performance in R. Bioinformatics, 2005, 21(20):3940-3941.

22. Yanuar A, Syahdi RR, Aryati WD, Parameter Optimization and Virtual Screening Indonesian Herbal Database as Human Immunodeficiency Virus -1 Integrase Inhibitor Using Autodock And Vina, International Journal of Applied Pharmaceutics, 2017, 9(Special Issue): 90-93.

23. Sotriffer C, Virtual Screening: Principles, Challenges, and Practical Guidelines, Wiley-Vch Verlag \& Co. KgaA, Weinheim, Germany, 2011, 115-119.

24. Lyne PD, Structure-based virtual screening: an overview, DDT, 2002, 7(20): 202. 